\title{
Irregular Prime Divisors of the Bernoulli Numbers
}

\author{
By Wells Johnson
}

\begin{abstract}
If $p$ is an irregular prime, $p<8000$, then the indices $2 n$ for which the Bernoulli quotients $B_{2 n} / 2 n$ are divisible by $p^{2}$ are completely characterized. In particular, it is always true that $2 n>p$ and that $B_{2 n} / 2 n \not \equiv\left(B_{2 n+p-1} / 2 n+p-1\right)\left(\bmod p^{2}\right)$ if $(p, 2 n)$ is an irregular pair. As a result, we obtain another verification that the cyclotomic invariants $\mu_{p}$ of Iwasawa all vanish for primes $p<8000$.
\end{abstract}

1. Introduction and Summary. Let $B_{n}$ denote the sequence of Bernoulli numbers in the "even-index" notation of [1]. If $B_{2 n}=P_{2 n} / Q_{2 n}$ with $\left(P_{2 n}, Q_{2 n}\right)=1$, then the prime factorization of the denominator $Q_{2 n}$ is given precisely by the von Staudt-Clausen theorem. The prime divisors of $P_{2 n}$, however, are more difficult to obtain. Their importance stems from the fact that, more than a century ago, Kummer proved that the Fermat equation $x^{p}+y^{p}=z^{p}$ has no integral solutions if $p$ is a regular prime, that is, one for which $p$ does not divide $P_{2} P_{4} P_{6} \cdots P_{p-3}$.

A rather old result, now commonly known as J. C. Adams' theorem (cf. [10, p. 261]), states that if $p$ is a prime not dividing $Q_{2 n}$ and $p^{e}$ divides $n$ for some $e \geqq 1$, then $p^{e}$ also divides $P_{2 n}$. Thus, given any prime power $p^{e}$ for $p>3, e \geqq 1$, there exist infinitely many Bernoulli numerators $P_{2 n}$ which are divisible by $p^{\circ}$. If we add the restriction that $(p, n)=1$, however, then the problem of determining when $p^{\circ}$ divides $P_{2 n}$ becomes more difficult. It turns out to be convenient to study the quotients $B_{2 n} / 2 n=P_{2 n} / 2 n Q_{2 n}$, which, when reduced, are $p$-integers if $p-1 \nmid 2 n$ by the theorems of von Staudt-Clausen and J. C. Adams.

The general problem, then, is to determine, for a given prime-power $p^{*}$, those indices, $2 n, p-1 \nmid 2 n$, for which $p^{e}$ divides the $p$-integer $B_{2 n} / 2 n$. It follows immediately from a congruence of Kummer that $p$ must be irregular, and that $p$ divides $B_{2 n} / 2 n$ if and only if $p$ divides $P_{2 n^{\prime}}$, where $2 n^{\prime}$ is the least positive residue of $2 n(\bmod p-1)$. This settles the case $e=1$. Moreover, we see that any irregular prime $p$ divides infinitely many Bernoulli numerators $P_{2 n}$ with $(p, n)=1$.

This paper reports on some computations done recently on the PDP-10 computer at Bowdoin College to investigate the case $e=2$. About fifty years ago, Pollaczek [9] noted that $37^{2}$ divides $B_{284} / 284$, showing that the case $e=2$ is possible. Montgomery [8] raised the question whether or not $p^{2}$ divides $P_{2 n}$ for $0<2 n<p-1$. Our computations show that the answer to this is negative for all irregular primes $p<8000$. Further, for the irregular primes $p<8000$, we can characterize precisely those indices $2 n$ for which $p^{2}$ divides $B_{2 n} / 2 n$. Our results show that the square of any irregular prime $p<8000$ divides infinitely many Bernoulli numerators $P_{2 n}$ with $(p, n)=1$. Finally, we compare some of our computations to those done earlier

Received April 9, 1973.

AMS (MOS) subject classifications (1970). Primary 10A40; Secondary 12 A35.

Key words and phrases. Bernoulli numbers, irregular primes, cyclotomic invariants.

Copyright (c) 1974, American Mathematical Society 
by Pollaczek [9] and discuss the important relationship of these results to the determination of the cyclotomic invariants $\mu_{p}$ of Iwasawa.

If $p$ is an irregular prime and $p$ divides $P_{2 n}$ for $0<2 n<p-1$, then we shall refer to $(p, 2 n)$ as an irregular pair. For a given irregular prime $p$, the number of such irregular pairs is called the index of irregularity of $p$.

2. The Congruences of Kummer. We state the fundamental congruences of Kummer (cf. [10, p. 266]), valid for $2 \leqq r+1 \leqq 2 n$ and primes $p$ for which $p-1 \nmid 2 n:$

$$
\sum_{s=0}^{r}(-1)^{s}\left(\begin{array}{l}
r \\
s
\end{array}\right) \frac{B_{2 n+s(p-1)}}{2 n+s(p-1)} \equiv 0\left(\bmod p^{r}\right) .
$$

For $r=1,2$ we obtain for $p-1 \nmid 2 n$ :

$$
\begin{gathered}
\frac{B_{2 n}}{2 n} \equiv \frac{B_{2 n+(p-1)}}{2 n+(p-1)}(\bmod p), \quad n \geqq 1, \\
\frac{B_{2 n}}{2 n}-2 \frac{B_{2 n+(p-1)}}{2 n+(p-1)}+\frac{B_{2 n+2(p-1)}}{2 n+2(p-1)} \equiv 0\left(\bmod p^{2}\right), \quad n \geqq 2 .
\end{gathered}
$$

An analysis of (1) gives the results stated in the previous section for the case $e=1$ of the general problem. We remark that the argument used here is essential for all known proofs of the existence of infinitely many irregular primes in certain arithmetic progressions (cf. [11], [2], [8], and [7]).

For the case $e=2$, we use Eq. (2). If $p^{2}$ divides $B_{2 n} / 2 n$, then as above, $\left(p, 2 n^{\prime}\right)$ must be an irregular pair, where $2 n^{\prime}$ is the least positive residue of $2 n(\bmod p-1)$. Also, given an irregular pair, $\left(p, 2 n^{\prime}\right)$, we define $A_{t}=B_{2 n^{\prime}+t(p-1)} / 2 n^{\prime}+t(p-1)$ for $t \geqq 0$. By (1), $A_{t} \equiv 0(\bmod p)$, so that we may define $a_{t}$ by the conditions $A_{t} \equiv$ $a_{t} p\left(\bmod p^{2}\right), 0 \leqq a_{t}<p$. Hence $p^{2}$ divides $A_{t}$ if and only if $a_{t}=0$. Since $B_{2}=\frac{1}{6}$, it follows that $n^{\prime}>1$. Equation (2) then implies that

$$
a_{t+2}-a_{t+1} \equiv a_{t+1}-a_{t}(\bmod p), \quad t \geqq 0,
$$

which gives

$$
a_{t}-a_{0} \equiv t\left(a_{1}-a_{0}\right)(\bmod p), \quad t \geqq 1 .
$$

Thus $p^{2}$ divides $B_{2 n} / 2 n$ if and only if $2 n=2 n^{\prime}+t(p-1)$, where $\left(p, 2 n^{\prime}\right)$ is an irregular pair, and where $t \geqq 0$ and $t$ satisfies the congruence

$$
-a_{0} \equiv t\left(a_{1}-a_{0}\right)(\bmod p) \text {. }
$$

Given an irregular pair $\left(p, 2 n^{\prime}\right)$, if it happens that $a_{1}=a_{0}$, then $a_{t}=a_{0}$ for all $t \geqq 1$. If $a_{0} \neq 0$, then $p^{2}$ divides no $B_{2 n} / 2 n$ with $2 n \equiv 2 n^{\prime}(\bmod p-1)$, but if $a_{0}=0$, then $p^{2}$ divides every $B_{2 n} / 2 n$ with $2 n \equiv 2 n^{\prime}(\bmod p-1)$. If $a_{1} \neq a_{0}$, however, then we can solve (3) for $t$ uniquely $(\bmod p)$. In this case, then, every interval of length $p^{2}-p$ contains exactly one index $2 n, 2 n \equiv 2 n^{\prime}(\bmod p-1)$, for which $p^{2}$ divides $B_{2 n} / 2 n$. The index $2 n$ is divisible by $p$ only when $t \equiv 2 n^{\prime}(\bmod p)$. Thus $p^{2}$ divides infinitely many Bernoulli numerators $P_{2 n}$ with $(p, n)=1$ if and only if, for some irregular pair $\left(p, 2 n^{\prime}\right)$, either (a) $a_{0}=a_{1}=0$ or (b) $a_{0} \neq a_{1}$ and the unique solution $t(\bmod p)$ to $(3)$ is not $2 n^{\prime}$. 
3. Computational Results. The values of $a_{0}$ and $a_{1}$ were computed for each of the 502 irregular pairs $(p, 2 n), p<8000$, previously reported by the author [5]. For all 502 pairs, it was found that $a_{0} \neq 0$, and that $a_{1} \neq a_{0}$ so that it was possible to solve (3) for $t(\bmod p)$. For no pair $(p, 2 n)$ did we ever obtain $t=2 n$. We thus have the following:

THEOREM. If $p$ is an irregular prime, $p<8000$, then

(A) $p^{2}$ does not divide any of the Bernoulli numerators $P_{2}, P_{4}, P_{6}, \cdots, P_{p-3}$.

(B) $B_{2 n} / 2 n \not \equiv\left(B_{2 n+(p-1)} / 2 n+(p-1)\right)\left(\bmod p^{2}\right)$ for all irregular pairs $(p, 2 n)$.

(C) Every interval of length $p^{2}-p$ contains exactly $i_{p}$ indices $2 n$ with $B_{2 n} / 2 n \equiv 0$ $\left(\bmod p^{2}\right)$, where $i_{p}$ is the index of irregularity of $p$. Moreover, for all of these, $(p, n)=1$, so that there exist infinitely many Bernoulli numerators $P_{2 n},(p, n)=1$, divisible by $p^{2}$.

For each irregular pair $(p, 2 n)$, the values of $a_{0}$ and $a_{1}$ were computed from the following equations of $\mathrm{E}$. Lehmer [6], valid for $p>5, p-1 \nmid 2 s-2$ :

$$
\sum_{r=1}^{[p / 4]}(p-4 r)^{2 s-1} \equiv\left(d_{2 s} B_{2 s} / 4 s\right)\left(\bmod p^{2}\right), \quad d_{2 s}=\left(2^{2 s}-1\right)\left(2^{2 s-1}+1\right) .
$$

For each irregular pair $(p, 2 n)$, we first tested for the invertibility of $c_{2 n}(\bmod p)$. For $c_{2 n} \neq \equiv 0(\bmod p)$, we next computed the sum $\left(\bmod p^{2}\right)$ in (4) with $2 s=2 n$, writing it in the form $e+f p, 0 \leqq e, f<p$. It was first checked that $e=0$, again verifying that indeed $(p, 2 n)$ is an irregular pair. Then $a_{0}$ was computed from the congruence $a_{0} \equiv 2 c_{2 n}{ }^{-1} f(\bmod p)$. The value of $a_{1}$ was found similarly, using (4) with $2 s=2 n+$ $p-1$. For only one irregular pair, $(1201,676)$, did $c_{2 n}$ fail to be invertible. For this pair, $d_{2 n} \not \equiv 0(\bmod p)$, so that we were able to compute the values of $a_{0}$ and $a_{1}$ from Eq. (5). After computing $t(\bmod p)$ from (3), we performed a final check by showing that the sum in $(4)$ or $(5)$ vanishes $\left(\bmod p^{2}\right)$ for $2 s=2 n+t(p-1)$. A partial table of our results is included at the end of this paper.

4. Pollaczek's Results and the Cyclotomic Invariants $\mu_{p}$ of Iwasawa. Pollaczek [9, p. 31] performed these computations some time ago for the three irregular primes $p<100$. He computed $\left(-B_{2 n} / n\right)$ rather than $\left(B_{2 n} / 2 n\right)\left(\bmod p^{2}\right)$, so that our values of $a_{0}$ and $a_{1}$ must be multiplied by -2 in order to make valid comparisons. The results agree for $p=37$ and also for $p=59$ after a transposition of Pollaczek's indices to correct his obvious inconsistency. For $p=67$, there seems to be an error in Pollaczek's value of $B_{62}{ }^{\prime}$, corresponding to our value of $a_{1}$. A direct computation of Eq. (4) negates his claim that $67^{2}$ divides $P_{190}$.

Iwasawa [3, p. 782] has shown that the cyclotomic invariant $\mu_{p}$, important in the theory of class numbers of cyclotomic fields, vanishes if $p$ is either a regular prime or an irregular prime for which $a_{0} \neq a_{1}$ for all irregular pairs $(p, 2 n)$. Iwasawa invoked the computations of Pollaczek to conclude that $\mu_{p}=0$ for all primes $p<100$. More recently, using other tests, Iwasawa and Sims [4] and the author [5] have shown that $\mu_{p}=0$ for all primes $p<8000$. The computations reported here give another verification that this is true. 


\section{TABLE}

\begin{tabular}{|c|c|c|c|c|}
\hline $\boldsymbol{p}$ & $2 n$ & $a_{0}$ & $a_{1}$ & \\
\hline $\begin{array}{r}37 \\
59 \\
67 \\
101 \\
103\end{array}$ & $\begin{array}{l}32 \\
44 \\
58 \\
68 \\
24\end{array}$ & $\begin{array}{r}1 \\
23 \\
43 \\
30 \\
98\end{array}$ & $\begin{array}{l}22 \\
49 \\
64 \\
72 \\
49\end{array}$ & 57 \\
\hline $\begin{array}{l}491 \\
491 \\
491 \\
523 \\
541\end{array}$ & $\begin{array}{r}292 \\
336 \\
338 \\
400 \\
86\end{array}$ & $\begin{array}{l}265 \\
225 \\
453 \\
413 \\
515\end{array}$ & $\begin{array}{l}230 \\
328 \\
437 \\
387 \\
185\end{array}$ & $\begin{array}{r}218 \\
260 \\
59 \\
36 \\
436\end{array}$ \\
\hline $\begin{array}{r}953 \\
971 \\
1061 \\
1091 \\
1117\end{array}$ & $\begin{array}{l}156 \\
166 \\
474 \\
888 \\
794\end{array}$ & $\begin{array}{r}827 \\
817 \\
87 \\
24 \\
210\end{array}$ & $\begin{array}{r}851 \\
561 \\
251 \\
781 \\
79\end{array}$ & $\begin{array}{r}720 \\
538 \\
1054 \\
85 \\
607\end{array}$ \\
\hline $\begin{array}{l}1997 \\
1997 \\
2003 \\
2003 \\
2017\end{array}$ & $\begin{array}{r}772 \\
1888 \\
60 \\
600 \\
1204\end{array}$ & $\begin{array}{r}508 \\
591 \\
1761 \\
1816 \\
1621\end{array}$ & $\begin{array}{r}163 \\
348 \\
319 \\
1656 \\
1547\end{array}$ & $\begin{array}{r}1136 \\
1531 \\
511 \\
1113 \\
1412\end{array}$ \\
\hline $\begin{array}{l}3989 \\
4001 \\
4003 \\
4003 \\
4003\end{array}$ & $\begin{array}{r}1936 \\
534 \\
82 \\
142 \\
2610\end{array}$ & $\begin{array}{r}933 \\
2447 \\
1757 \\
430 \\
2010\end{array}$ & $\begin{array}{r}1306 \\
2861 \\
3792 \\
85 \\
3594\end{array}$ & $\begin{array}{r}3794 \\
3019 \\
784 \\
3018 \\
2258\end{array}$ \\
\hline $\begin{array}{l}5939 \\
5939 \\
5953 \\
6007 \\
6011\end{array}$ & $\begin{array}{r}342 \\
5014 \\
3274 \\
912 \\
5870\end{array}$ & $\begin{array}{l}3660 \\
3488 \\
1007 \\
4702 \\
5292\end{array}$ & $\begin{array}{r}124 \\
4069 \\
3675 \\
3459 \\
399\end{array}$ & $\begin{array}{l}3031 \\
5749 \\
2068 \\
4445 \\
4232\end{array}$ \\
\hline $\begin{array}{l}7937 \\
7949 \\
7949 \\
7951 \\
7963\end{array}$ & $\begin{array}{l}3980 \\
2506 \\
3436 \\
4328 \\
4748\end{array}$ & $\begin{array}{l}3192 \\
3876 \\
7398 \\
5767 \\
5527\end{array}$ & $\begin{array}{l}5703 \\
5215 \\
2031 \\
6327 \\
5570\end{array}$ & $\begin{array}{r}4503 \\
2906 \\
2263 \\
799 \\
3390\end{array}$ \\
\hline
\end{tabular}

$$
\begin{gathered}
2 n+t(p-1) \\
284 \\
914 \\
3292 \\
5768 \\
228
\end{gathered}
$$

107112

127736

29248

19192

235526

685596

522026

1117714

93538

678206

2268228

3057764

1023082

2228826

2847796

15132408

12076534

3137650

12078178

9039126

17998420

34142576

12312010

26697582

25440190

35739788

23099394

17989760

6356378

26995928

Department of Mathematics

Bowdoin College

Brunswick, Maine 04011

1. Z. I. BoREvǐ̌ \& I. R. SAFAREVIČ, Number Theory, "Nauka", Moscow, 1964; English transl., Pure and Appl. Math., vol. 20, Academic Press, New York, 1966. MR 30 \#1080; MR 33 \#4001.

2. L. Carlitz, "Note on irregular primes," Proc. Amer. Math. Soc., v. 5, 1954, pp. 329-331. MR 15, 778.

3. K. IWASAWA, "On some invariants of cyclotomic fields," Amer. J. Math., v. 80, 1958, pp. 773-783; erratum, ibid., v. 81, 1959, p. 280. MR 23 \#A1631.

4. K. IWASAWA \& C. SIMS, "Computation of invariants in the theory of cyclotomic fields," J. Math. Soc. Japan, v. 18, 1966, pp. 86-96. MR $34 \# 2560$.

5. W. Johnson, "On the vanishing of the Iwasawa invariant $\mu_{p}$ for $p<8000$," Math. Comp., v. 27, 1973, pp. 387-396. 
6. E. LEHMER, "On congruences involving Bernoulli numbers and the quotients of Fermat and Wilson," Ann. of Math., (2), v. 39, 1938, pp. 350-360.

7. T. MetsïnkyLÄ, "Note on the distribution of irregular primes," Ann. Acad. Sci. Fenn. Ser. A.I., v. $492,1971,7$ pp. $\mathrm{MR} 43$ \#168.

8. H. L. MONTGOMERY, "Distribution of irregular primes," Illinois J. Math., v. 9, 1965, pp. 553-558. MR 31 \#5861.

9. F. Pollaczer, "Über die irregulären Kreiskörper der l-ten und $l^{2}$-ten Einheitswurzeln," Math. Z., No. 21, 1924, pp. 1-38.

10. J. Uspensix \& M. Heaslet, Elementary Number Theory, McGraw-Hill, New York, 1939. MR 1, 38.

11. H. S. VANDIVER, "Is there an infinity of regular primes?," Scripta Math., v. 21, 1955, pp. $306-309$. 